\title{
Wireless body area network's application for motion detection based on Android smartwatch
}

\author{
Eppy Yundra ${ }^{1, *}$, Arif Widodo ${ }^{1}$, Pradini Puspitaningayu ${ }^{1}$, and Unit Three Kartini ${ }^{1}$ \\ ${ }^{1}$ Universitas Negeri Surabaya, Department of Electrical Engineering, Faculty of Engineering, Indonesia
}

\begin{abstract}
Short-range, wireless communications in the vicinity of, or inside, a human body (but not limited to humans) are specified in this standard. It uses existing industrial scientific medical (ISM) bands as well as frequency bands approved by national medical and/or regulatory authorities. These sensors provide accurate and reliable information about the situation or activity occurring at any time. This high responsiveness of these sensors has made lives easy and safe. This article will perform an investigation on the WBAN's application for motion detection based on android smartwatch. The main problem is how to the sensors that attached on the human body can send of data via Bluetooth and read the motion and orientation of the body and display in the graph form. However, if the sensors do not match, the result of output display inappropriate. This article proposed method by using accelerometer and gyroscope sensors which are integrated with smartwatch to overcome the main problem. This application will display results in the form of numeric values and then convert in graph form. These results have proven that WBAN's application for motion sensing can be used for detection of motion and orientation via Bluetooth based on android smartwatch.
\end{abstract}

\section{Introduction}

Nowadays, the need of quantity and quality of health service that fast continue to increase. It is caused by the increasing amount of elderly who followed by chronic health problems. Moreover, people now become more aware of health, so they require more service to prevent, monitor, and even cure the diseases. The advances in wireless communication technologies and smart sensors, a variety of e-health applications have been realized to provide efficient healthcare services, and that is how wireless body area networks (WBANs) take part.

The several efforts in WBAN has significantly increased in recent years motivated by the attracting applications that can be enabled by this technology is a multitude of fields [1]. Open issues and challenges within each area are also explored as a source of inspiration towards future developments in wireless body area networks (WBAN). The IEEE 802.15 has standardized this technology with low power, low-cost and low bit rate performance [2].

The current WBAN technology which very close to daily life is built in a smartphone. Many hand-held devices have sensors, even without the user's knowing. Accelerometer and gyroscope is one of the most included sensors.

At the same time, the development smartphones technology and its platform also support the mobility of WBAN technology. The system utilizes the user's own smartphone for data processing, and the built-in communications can be used to raise an alarm if a heart attack is detected. This is managed by an application for Android smartphones that has been developed for this system. The good functionality of the system was confirmed in three real-life user case scenarios [3]. The processing of the ECG and ACC data is performed by the custom-developed software installed on PC. All recorded information is uploaded to a purposelydesigned medical web server for the storage and display as a web page for authorized doctors or patient's family. The system was tested on 10 healthy volunteers. Each of them was monitored during common daily activities [4]. Therefore, accelerometer can also be used in a more complex sensor networks to give more information for health monitoring [5,6]. However, all of the abovementioned models only consider for each sensors but they do not consider combine sensors for motion detection.

The authors of [7] present an accelerometer assisted transmission power control (AA-TPC) scheme which exploits the periodic fluctuations of link qualities to improve the transmission energy efficiency. Consider the relationship between link quality and body movement, AA-TPC makes transmissions at ideal channel points that are identified by using the local accelerometer. Android platform is chosen by many researchers for its popularity and open-source characteristic, so the developers can freely expand the applications for many purposes. However, the specific transmission power is then determined by the feedback information from the receiver.

\footnotetext{
* Corresponding author: eppyyundra@unesa.ac.id
} 
The author of [8] present a model the channel access for star network that analyze the overall performance of IEEE 802.15.4 MAC to reduce probability of going to next back off stage which can increase probability success packet transmission with adjustment delay at first CCA and second CCA if channel find in busy condition also including addition third CCA to reduce collision in order for improve performance networks. However, only implementation of super frame duration system.

This article will perform an investigation on the WBAN's application for motion detection based on android smartwatch. The main problem is how to the sensors that attached on the human body can send of data via Bluetooth and read the motion and orientation of the body and display in the graph form. However, if the sensors do not match, the result of output display inappropriate. The major contribution of this article is to propose method by using accelerometer and gyroscope sensors which are integrated with smartwatch to overcome the main problem. This application will display results in the form of numeric values and then convert in graph form.

\section{Wireless body area networks}

A health-care system that promises the continuous and reliable gathering and objective analysis of physiological and behavioral aspects of a patient, and delivers this information to physicians, have been the goal of body area networks (BANs). BANs can tackle health-care monitoring and delivery challenges by wireless technology and mobile and cloud computing through the use of independent sensors and actuators attached to the body. BANs can accomplish what human-computer interaction aims for, i.e., the design of technologies that are flexible to human needs [9].

In a wireless BAN, the nodes or sensors are placed on the body or on everyday clothing. These sensors that create an interface to humans have ultra-low power connectivity, which allow them to be portable and measure vital signs outside a hospital or other healthcare settings. The data can be accessed by the user, hospital, doctor or physician, and any other authorised users. Actually, WBANs is not only can be applied for medical purpose but also for entertainment, sports, military, and other applications [10]. The figure below is an illustration of a wireless body area network.

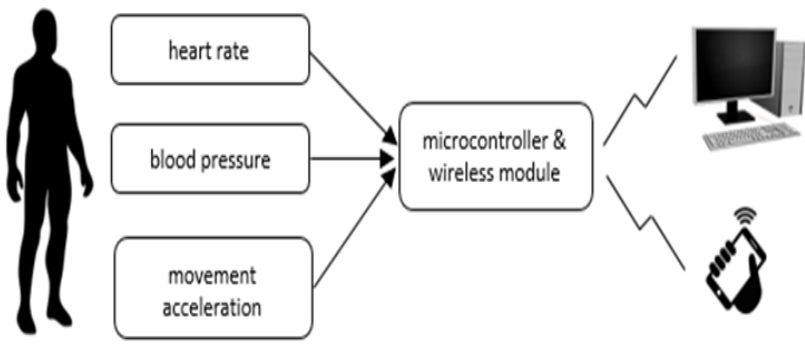

Fig. 1. Simplified WBAN network architecture.

Short-range, wireless communications in the vicinity of, or inside, a human body (but not limited to humans) are specified in this standard. It uses existing industrial scientific medical (ISM) bands as well as frequency bands approved by national medical and/or regulatory authorities. Support for quality of service (QoS), extremely low power, and data rates up to $10 \mathrm{Mbps}$ is required while simultaneously complying with strict non-interference guidelines where needed [11].

Wireless Body Area Sensor Network (WBASN) is a network of implanted biosensors on/inside the human body for remote health monitoring. Considering the need for a reliable health care information system, security of WBASN is key to effective remote health monitoring of users and the development of proper mobile-health (ehealth) care delivery system. The concept of WBASN, the applications, security challenges and a review on security as it pertains to WBASN [12].

At the medical network, the data can be used for further analysis and/or diagnosis to the monitored symptoms of the user. A typical health monitoring system consists of a network of wearable or implanted sensors that constantly monitor physiological parameters [13]. Collected data is relayed using existing wireless communication protocols to a base station for additional processing [14]. As mentioned earlier, WBAN application can be very wide. For medical purpose only, there are various sensors which can be used such as heart rate sensor, ECG, EEG, EMG, blood pressure, blood oxygen saturation, accelerometer, gyroscope, magnetometer, and many more. In the WBAN for running time to take the data maybe can consider for allocate length of guaranteed time slot (GTS). The assigning adjustable length of GTS slot based on the length of packet and also deciding the precise time for the GTS starting time (GTSstart) and the GTS length (GTSlength) for star topology must be appropriate [15].

Accelerometer and gyroscope are used to monitor the acceleration of the user's movement and the orientation respectively. The data can be continuously changed during the sensors are worn and the user is moving.

\section{System design}

The design of this system is actually included in the smartphone. However, the general WBAN architecture is illustrated in figure 2. The development process is done by following the Android development process.

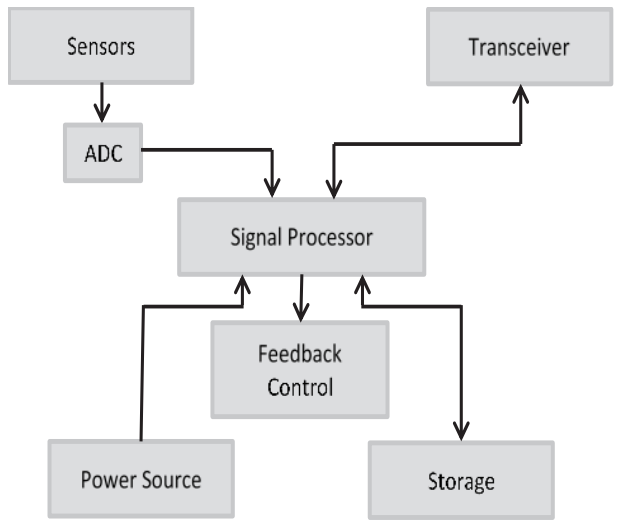

Fig. 2. WBAN Architecture. 
The data collection of process diagram is shown in Figure 3 where it begins by programming the application, installing it to the smartphone, moving it so that the sensor can detect acceleration and orientation, the application then reads and represents data into the graph that can keep changing as long as the smartphone is being moved.

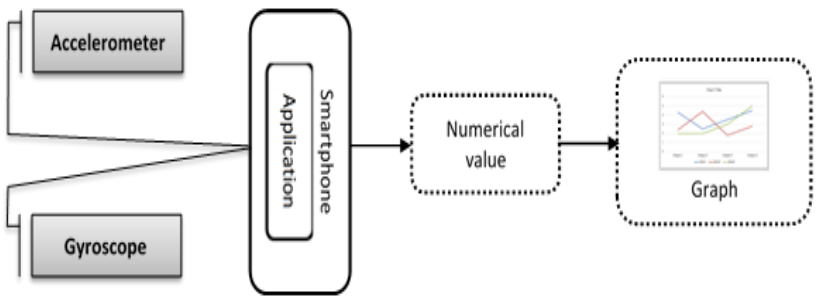

Fig. 3. General system design.

\section{Result and discussion}

The results of testing have been done several steps and all the sensor functions present in the smartwatch work well. The typical smartwatch will include one or a combination of the feature [16]. In this case, focus on accelerometer and gyroscope sensors. The first step, application is able to read random movement acceleration and orientation of the user when the smartphone is being held. Then the numerical data can be collected and converted into a graph as shown in the figure 4 and 5 respectively. For example, when the user shakes the smartphone, the movement graph can be shown on the application.

\section{Sensor2}

$$
\begin{aligned}
& \text { Acceleration } x \\
& :-0.29688102 \\
& \text { Acceleration } y \\
& : 2.9442697 \\
& \text { Acceleration } z \\
& : 9.648034 \\
& \text { Gyroscope } X \\
& : 0.007456851 \\
& \text { Gryoscope } y \\
& : 0.017044231 \\
& \text { Gyroscope } z \\
& :-0.04580637 \\
& \text { Linear Acceleration } x \\
& :-0.38081568 \\
& \text { Linear Acceleration } y
\end{aligned}
$$

\section{$: 0.3057146$}

Linear Acceleration 2

\section{$: 0.20338726$}

Fig. 4. Numerical value.

The next step, application process is developed for smartwatch. When smartwatch is implemented and the application is run, a change of numerical value of the accelerometer appears as shown in Figure 6.

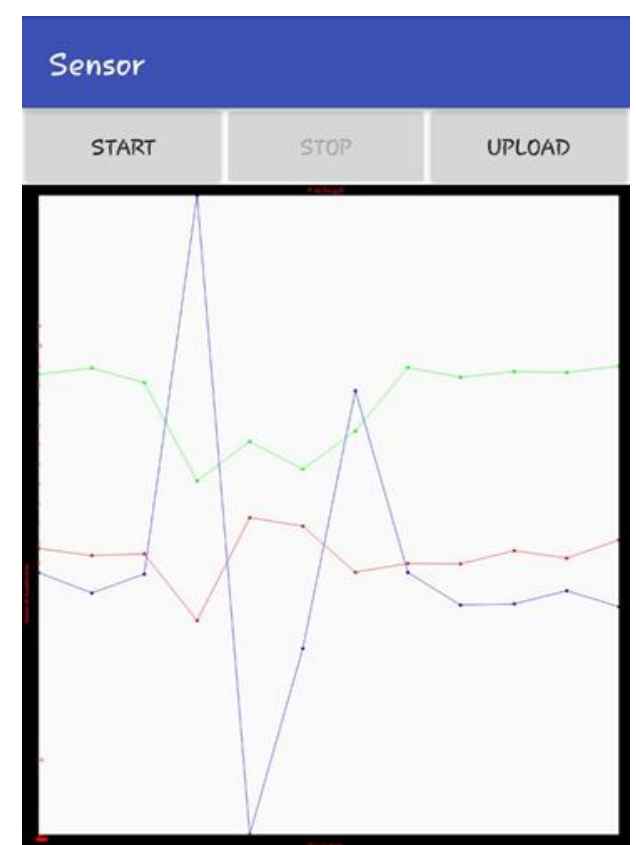

Fig. 5. The graph of the movement.

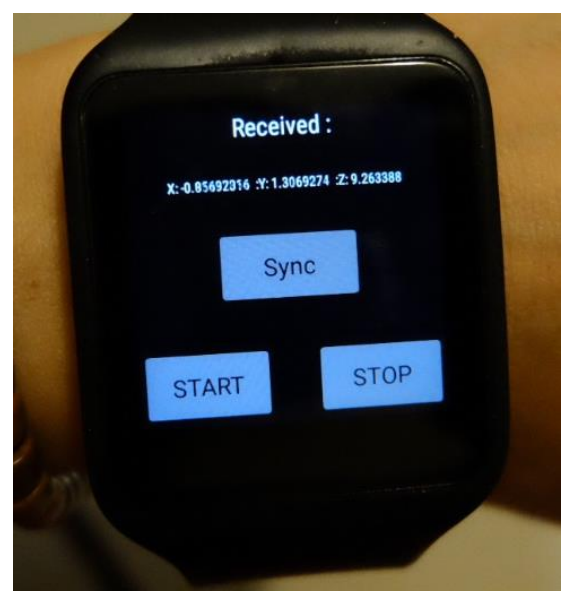

Fig. 6. The numerical value of accelerometer on smartwatch.

Figure 6 shows the application is implemented on smartwatch. As seen consisting of three keys namely sync, start and stop are used to sync with the smartphone, start the direction and motion detection and end of the application, respectively.

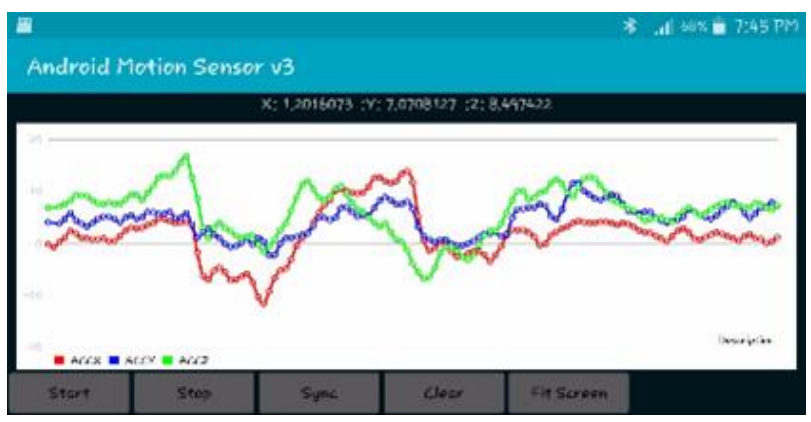

Fig. 7. The graph of smartwatch. 
Figure 7 shows the result that data from smartwatch is successfully sent via Bluetooth to smartphone. The result of motion detection and direction can be read accelerometer and gyroscope sensors in the form of numeric value then it is converted in to graph form by smartphone.

\section{Conclusions}

This application has been displayed results in the form of numeric values and then convert in graph form. These results have proven that sensors can transmit data with Bluetooth properly and sensors that put on human body using WBAN's application for motion sensing can be used for detection of motion and orientation via Bluetooth based on android smartwatch.

\section{References}

1. R Cavallari. A Survey on Wireless Body Area Networks: Technologies and Design Challenges. IEEE Communication Surveys \& Tutorials. (2014)

2. S Movassaghi Wireless Body Area Networks: A Survey. IEEE Communications Surveys \& Tutorials. (2013)

3. G Wogast Wireless Body Area Network for Heart Attack Detection. IEEE Antennas \& Propagation Magazine. (2016).

4. E Kantoch, Wireless Body Area Network System based on ECG and Accelerometer Pattern. IEEE Computing in Cardiology (2011)

5. A M Nia. Energy-Efficient Long-term Continuous Personal Health Monitoring. IEEE Transactions on Multi-Scale Computing Systems ( 2015)

6. H Rezaie, M Ghassemian. An Adaptive Algorithm to Improve Energy Efficiency in Wearable Activity Recognition Systems. IEEE Sensors J. (2016)

7. W Zang, S Zhang. An Accelerometer-Assisted Transmission Power Control Solution for EnergyEfficient Communications in WBAN, IEEE J. on Selected Areas in Communications - Series on Green Communications and Networking (Issue 2) (2015)

8. E. Yundra at al. Study of Adjustment Delay Scheme on IEEE 802.15.4 Networks at Beacon Enabled Mode. The 2nd Annual Applied Science and Engineering Conference, Bandung 2017. 288 (2017)

9. B Johny, A Anpalagan. Body Area Sensor Networks: Requirements, Operations, and Challenges. IEEE Potentials Magazine March/April (2014).

10. F Hu A Human Body Posture Recognition Algorithm Based on BP Neural Network for WBANs. China Communications, 13 (2016)

11. IEEE 802.15.6 standards. Part 15.6: “ireless Body Area Networks. IEEE Standard for Local and metropolitan area networks (2012)
12. A Sangwan, P Battacharya. Wireless Body Sensor Networks: A Review. Int. J. of Hybrid Information Technology (2015)

13. A Rangarajan. Emerging Trends in Healthcare Adoption of Wireless Body Area Networks. AAMI Biomedical Instrumentation \& Technology (2016)

14. M Ghamari A Survey on Wireless Body Area Networks for eHealthcare Systems in Residential Environments. Sensors MDPI J. (2016)

15. B-H Lee, Eppy Yundra, $\mathrm{H}-\mathrm{K} \mathrm{Wu}$ and $\mathrm{M}$ Udin Harun Al Rasyid. Analysis of superframe duration adjustment scheme for IEEE 802.15.4 networks. EURASIP $\mathrm{J}$ on Wireless Communication and Networking. 103. (2015)

16. R Lutze, K Waldhör, The Application Architecture of Smartwatch Apps-Analysis, Principles of Design and Organization. Gesellschaft für, Informatik, Bonn. (2016) 